\section{Das Verhalten der Halogene gegen Röntgenstrahlen.}

Von E. Sehrwald, Stabsarzt in Freiburg i. B.

Bei der Bedeutung, die einem Theil der Halogene für den Aufbau der thierischen und pflanzlichen Gewebe zukommt, schien es von Interesse festzustellen, wie sich diese Elemente den Röntgenstrahlen gegenüber verhalten.

Von vornherein ist man wohl geneigt, vorwiegend oder ausschliesslich die Metalle als die Stoffe zu betrachten, die für die X-Stralılen undurchgängig sind. Eine nebensächliche Beobachtung machte es mir sellr wahrscheinlich, dass auch die Halogene, also das Jod, Brom, Chlor und Fluor, die gleiche Eigenthümlichkeit, bei der Beleuchtung mit X-Strahlen intensive Schatten zu werfen, besitzen müssten. Die Untersuchung hat diese Vermuthung im vollsten Maasse bestätigt. Zugleich führte sie aber auch zu dem theoretischen Schluss, dass es eine Reihe wasserklarer und dem Licht gegenüber völlig durchsiclitiger Flüssigkeiten geben müsse, die sich für Röntgenstrahlen in ganz ähnlicher Weise, wie Metalle, undurchlässig zeigen müssten. Und in der That liess sich auch für eine Anzahl Flüssigkeiten dieses höchst überraschende, theoretisch postulirte Verhalten feststellen.

Auf einem Actinogramm antiseptischer Pulver und Lösungen') war mir aufgefallen, welch' unerwartet starken Schatten das Jodoform schon in sehr dünner Schicht und ill sehr kleinen Klüınpehen geworfen hatte. Bei dor chemischen Zusammensetzung des Jodoforms aus einem Atom Kollenstoff, einem Atom Wasserstoff und drei Atomen Jod lag die Vermuthung sehr nahe, dass nur die Jodcomponente es sein könne, die für die Röntgenstrahlen so undurchsichtig ist. Der Wasserstoff konnte schon seiner geringen Menge wegen kaum in Betracht kommen, denn er macht nur 0,25\% der Verbindung aus. Dies bestätigt zudem auch der direkte Versuch. Durchleuchtet man eine $6 \mathrm{~mm}$ dicke Schicht destillirten Wassers mit X-Strahlen, so erhält man nur einen sehr schwachen Schatten von der Flüssigkeitsschicht: zugleich ein Beweis, dass ausser dem Wasserstoff auch der im Wasser gewissermaassen verflüssigte Sauerstoff für Röntgenstrahlen fast völlig durchlässig ist.

Ebenso wenig. konnte die Scliattenbildung des Jodoforms in dem einen Atom Kohlenstoff ihren Grund haben. Der Kohlenstoff beträgt zwar 3,04\% in der Verbindung, kann aber auch nicht die Ursache der starken Schattenbildung sein, da experimentell nachgewiesen ist, dass der Diamant auch in dicker Schicht kaum eine Andeutung eines Schattens giebt, bekanntlich eines der sichersten Mittel, den Diamant von Imitationen zu unterscheiden.2) Aber auch die amorphe, schwarze Kohle zeigt das ganz gleiche Verhalten. Ich habe ein Glasröhrchen mit dicht zusammen gepresstem Lampenruss gefüllt, der durch unvollkommene Verbrennung von Petroleum gewonnen war. Diese dichte, schwarze Masse hat kaum die geringste Andeutung eines Schattens ergeben. Ebenso ist reinstes Terpentinöl $\mathrm{C}_{10} \mathrm{H}_{16}$ trotz des hohen Kohlengehalts fast völlig durchstrahlbar. Es bleibt also nur das Jod als der undurchlässige Körper übrig.

Ein orientirender Versuch ergab auch sofort, dass Jodblättchen in $1-2 \mathrm{~mm}$ dicker Lage noch nach 20 Minuten kaum eine Spur von X-Strahlen durchgelassen hatten. Selbst ganz vereinzelte Jodflitterchen hatten einen starken, schwarzen Schatten gegeben, und auch das Pergamentpapier, mit dem das Jod eingehüllt und das vom Jod leicht gebräunt war, hatte sich als schwache Schattenlinie abgebildet, während es sonst bei dieser Expositionsdauer im Bild überhaupt nicht sichtbar wird.

Dieses Verhalten eines Nichtmetalls gegen Röntgenstrahlen ist rein chemisch betrachtet sehr auffallend. Physikalisch erscheint diese Undurchlässigkeit auf den ersten Blick vielleicht weniger überraschend, da das Jod ja das Aussehen feiner Metallblättchen besitzt.

Es drängte sich nun die Frage auf, ob das Jod hier eine Ausnahmestellung einnimmt, oder ob sich die ganze chemische Gruppe der Halogene analog verhält. Ich stellte daher eine Reihe vergleichender Versuche an.

Die zu untersuchenden Stoffe wurden in gleich weite Röhrchen von bleifreiem Glas gebracht, da bleihaltiges Glas die X-Strahlen fast ganz absorbirt. Die Röhrchen waren im Innern $6 \mathrm{~mm}$ weit, ganz mit der Substanz angefüllt und mit Korkstöpseln fest verschlossen. Nur das Brom wurde wegen seiner starken Einwirkung auf organische Stoffe eingeschmolzen. Unı sämmtliche Gläser in ihren analogen Abschnitten gleich stark von den Strahlell bescheinen zu lassen, wurden sie nicht, wie man es sonst wohl gemacht hat, in Reihen angeordnet, da die entfernteren Gläser dann

1) Veroffentlichungen aus dem Gebiet des Militärsanitätswesens, Heft 10, S. 20 und Tafel XIV, 1896

2) C. Doelter, Die Unterscheidung der Edelsteine vermittels der X-Strahlen. Naturw. Rundschau 1896, Bd. XI, S. 277. entsprechend dem Quadrat ihrer grösseren Entfernung schwäclier beschienen werdell, sondern in einem Kreis, genau über dessen Mittelpunkt das Centrum der Vacuumröhre in $191 / 2 \mathrm{~cm}$ Abstand eingestellt war. Der untere kreisförmige Durchmesser der Vacuumröhre betrug $2 \mathrm{~cm}$. Eine Blende wurde nicht angewandt. Die Röhre war mit der Quecksilberluftpumpe verbunden. Ihr Druck schwankte zwischen 1 und $1 \frac{1}{2}$ Millionstel Atmosphäre. Die Bestrahlung dauerte $32 \frac{1}{2}$ Minuten. Bei den späteren Versuchen wurden zugeschmolzene Vacuumröhren verwandt und die Expositionszeit auf 7 und 21 Minuten verkürzt. Die Röhren waren von Herrn Prof. Zehnder in Freiburg i. B., einem Schüler Röntgen's, selbst hergestellt und nebst dem gesammten Apparat mir in liebenswürdigster Weise zur Verfügung gestellt worden. Die Bilder besitzen im Original eine ganz ungewöhnliche Schärfe und Klarheit.

Das erste Gläschen enthielt fein vertheiltes Jod; das zweite reines, flüssiges Brom, eingeschmolzen, und das dritte Chlorwasser von $0,5 \%$ Chlorgehalt. Das Gläschen mit Jod hat einen tief schwarzen Schatten gegeben, der sich von dem Schatten des Messings z. B. in nichts unterscheidet. Fin Blick auf die anderen Gläschen zeigte aber sofort, dass das Verhalten des Jods kein isolirtes in der Halogengruppe ist. Das Brom hat, obgleich es flüssig ist, einen ebenso intensiven und absoluten Schatten geworfen, wie das Jod. Das Bromgläschen war nur zu $2 / 3$ mit Brom gefüllt und mit der zugeschmolzenen Spitze wenige Millimeter höher gelagert, als der Boden des Gläschens. Die Flüssigkeitssäule verdünnt sich also in der Röhre gegen die Spitze mehr und mehr. Trotzdem haben auch die dünnsten Flüssigkeitsschichten noch einen fast absoluten Schatten ergeben; und auch das kleine Bromtröpfehen in der Spitze selbst und ein noch kleineres Tröpfchen zwischen der Spitze und dem Ende der Flüssigkeitssäule hat sich trotz der langen Expositionsdauer tief schwarz abgebildet. Das Brom scheint also trotz des $1 / 3$ miedrigeren Atomgewichtes fast ebenso undurchlässig zu sein, wie das Jod.

Das Chlor stand mir nur als 0,5\% iges Chlorwasser zur Verfügung. Ein direkter Vergleich mit den zwei anderen $200 \mathrm{Mal}$ concentrirteren Stoffen ist daher nicht möglich. Immerhin hat djese sehr schwache, wasserklare Lösung einen deutlichen Schatten gegeben, der auch gegen den Stöpsel zu, wo die Flüssigkeit bis zu den dünnsten Lagen sich auskeilt, noch deutlich ist. Auch die minimale Flüssigkeitsmenge, die an der Unterfläche des Korkes haftet, hat sich noch als scharfer Ringschatten abgebildet. Dieser Schatten ist sehr ausgesprochen dunkler, als der des reinen Wassers.

Um zu sehen, ob es das Atom der Halogene an und für sich ist, das die Röntgenstrahlen nicht passiren lässt, oder ob diese Undurchstrahlbarkeit nur eine Folge der Anordnung der Atome im Molekül des Jods, Broms und Chlors ist, habe ich von diesen Elementen analoge Verbindungen aus der anorganischen und organischen Natur untersucht.

Aus der anorganischen Reihe habe icl das Kalisalz verwandt. Alle drei Salze, das Jodkali, Bromkali und Chlorkali waren fein gepulvert. Sie haben sämmtlich eine sehr starke Schattenbildung ergeben. Der Schatten des Jod- und Bromkalis ist absolut schwarz, der des Chlorkalis eine Spur heller. Infolge dessen sind beim Chlorkali wenigstens an den Rändern des Gläschens noch die einzelnen Salzkörnchen zum Theil zu erkennen. Jedenfalls zeigt dieser Versuch, dass auch das Chlor einen ausgesprochenen Schatten wirft. Hingegen ist aus diesem Befund nicht ohne weiteres der Schluss erlaubt, dass das Chlor leichter durchgängig sei, als das Jod und Brom. Denu das Chlorkali enthält nur 47,65\% des Halogens, während das Bromkali $67,22 \%$ und das Jodkali 76,50\%, also ganz wesentlich mehr enthalt.

Wie viel von dem Schatten bei diesen drei Salzen auf die Metallcomponente, das Kali entfällt, ist annähernd aus einem mit Cyankalium gefüllten Gläschen zu ersehen. Das Cyankalium enthält fast so viel Kalium, wie das Chlorkali, nämlich $60 \%$. Im Cyan, CN, ist die Kohle, wie oben gezeigt wurde, fast ganz durchlässig für X-Strahlen. Nach der guten Durchlässigkeit auch grösserer Schichten atmosphärischer Luft zu schliessen, wird man das auch für den Stickstoff annehmen dürfen. Um hierüber einen sichereren Anhalt zu gewinnen, habe ich den Stickstoff in chemisch gebundener Form noch direkt untersucht und zwar als hoch concentrirte, wässrige Ammoniaklösung. Diese giebt einen Schatten, der nur wenig stärker ist, als der des reinen Wassers. Der Stickstoff in flüssigen Verbindungen ist daher zwar nicht absolut, aber doch in hohem Grade durchlässig. Ein weiterer Versuch mit einer sehr stickstoffreichen. Flüssigkeit, mit rother, rauchender Salpetersäure, gab nicht den gewünschten Aufschluss, da die Säure in bleihaltiges Glas eingeschmolzen war, und das Glas an sich schon so undurchlässig war, dass die Intensität des Flüssigkeitsschattens dagegen ganz zurücktrat.

Man kann daher die Schattenbildung des Cyankalis fast ganz auf Rechnung des Kaliums setzen. Da das Chlorkalium trotz 
eines etwas geringeren Kaliumgehalts doch einen sehr viel intensiveren Schatten wirft, muss man schliessen, dass auch das Chloratom für die X-Strahlen schwer passirbar ist.

Viel deutlicher ergiebt sich das noch aus dem Verhalten organischer Halogenverbindungen. Als Versuchsobjecte wurden die Methanderivate mit drei Haloidatomen benutzt. Von diesen ist das Jodoform pulverförmig, das Bromoform und Chloroform hingegen sind völlig wasserklare Flüssigkeiten und dem Licht gegenüber vollständig durchsichtig. Das Jodoform enthält $96,75 \%$ Jod, das Bromoform 94,86\% Brom und das Chloroform 89,12\% Chlor. Das Chloroform kann daher als verflüssigtes Chlor betrachtet werden, dem noch $10 \%$ Kohle nebst Wasserstoff beigemischt sind. Alle drei Stoffe mussten daher fast das gleiche Verhalten wie die reinen Halogene zeigen, da das eine Atom Kohlen- und Wasserstoff für die Durchlässigkeit nicht in Betracht kommt. Von vornherein erschien es freilich sehr wenig wahrscheinlich, dass zwei wasserklare Flüssigkeiten annähernd gleich dunkle Schatten werfen sollten, wie das undurchsichtige Brom oder Jod. Trotzdem verlangt die chemische Zusammensetzung dieser Stoffe eine fast völlige Undurchstrahlbarkeit für X-Strahlen. Und in der That stand das Ergebniss des Versuches in vollstem Einklang mit der theoretischen Forderung.

Das Jodoform hat einen fast absoluten Schatten geworfen, der sich von dem des reinen Jods kaum unterscheidet. Aber auch das wasserklare Bromoform hat sich den XStrahlen gegenüber fast ganz undurchlässig erwiesen und unterscheidet sich insofern kaum vom reinen Brom. Und ebenso hat sich auch das Chloroform tief schwarz abgebildet. Dass beim Bromoform und Chloroform dieses überraschende Resultat nicht etwa durch stark metallhaltiges Glas vorgetäuscht ist, sieht man an den Rändern de freien Oeffnung der Gläser, die ebenso hell und zart sich abgebildet haben, wie ein ganz leeres, nach Röntgen photographirtes Glas.

Eine genauere Vergleichung der drei Schattenbilder ergiebt, dass der Schatten des Chloroforms ein wenig heller ist, als das Bild der zwei anderen Stoffe. Das spricht aber zunächst noch nicht für eine grössere Durchlässigkeit des Chloratoms, sondern erklärt sich aus dem geringeren Haloidgehalt des Chloroforms, der nur 89,12\% beträgt gegenüber $96,75 \%$ beim Jodoform. Dass auch sehr dünne Chloroformschichten noch deutlichen Schatten geben, sieht man an den geringen Chloroformmengen, die zwischen Kork und Gläschen noch haften und deutlich als dunklere Schleier im Bild zu erkennen sind.

Um dies überraschende Resultat beim Chloroform und Bromoform absolut sicher zu stellen, habe ich vom Chloroform im ganzen vier, vom Bromoform drei Aufnahmen gemacht und das Chloroform dabei aus ganz verschiedenen Quellen bezogen. Stets war aber der Frfolg absolut der gleiche. Um auch dünnere Schichten der beiden Flüssigkeiten prüfen zu können, habe ich beide auch noch in Capillarröhrchen eingeschmolzen untersucht. Auch in capillarer Schicht ist der Schatten des Chloroforms noch dunkelgrau, aber zugleich sehr deutlich heller, als der des Bromoforms. Der Bromoformschatten ist aber auch in der dünnsten Schicht noch absolut schwarz und in nichts zu unterscheiden von dem Schatten eines gleich dicken Messingstiftes.

Das wasserklare Bromoform scheint also ein fast absolutes Filter für Röntgenstrahlen darzustellen und würde geeignet sein, aus Bündeln von Strahlen verschiedener Qualität die Röntgenstrahlen völlig zurück zu halten, während es z. B. die Lichtstrahlen ebenso vollständig hindurchlässt. Ein ganz ăhnliches, nur etwas weniger dichtes Filter würde das Chloroform darstellen.
Es war zu erwarten, dass gleich dem Chloroform und Bromoform auch andere chemisch nahe stehende organische Haloidverbindungen für die X-Strahlen ein starkes Hinderniss bieten würden. Ersetzt man in dem Chloroform das letzte Wasserstoffatom auch noch durch Chlor, so erhält man den flüssigen Chlorkohlenstoff, $\mathrm{C} \mathrm{Cl}_{4}$, mit vier Chloratomen oder $92,20 \%$ Chlor, während das Chloroform nur 89,12\% enthält. Auch der Chlorkohlenstoff ist eine wasserklare Flüssigkeit. Dem höheren Chlorgehalt entsprechend müsste sein Schatten noch etwas dunkler sein, als der des Chloroforms. Das ist thatsächlich auch der Fall. Besonders deutlich wird das beim Bestrahlen in capillarer Schicht. Hier ist der Schatten des Chlorkohlenstoffs ausgesprochen dunkler, als der des Chloroforms, aber immer noch erheblich heller, als der des Bromoforms.

Eine weiterє Bestätigung der starken Undurchlässigkeit auch des Chlors gab die photographische Untersuchung des Chloralhydrats. Das Chloralhydrat als dreifach gechlortes Aldehyd, $\left.\underset{\mathrm{H}}{\mathrm{CCl}_{3}}\right\} \mathrm{CO}+\mathrm{H}_{2} \mathrm{O}$, enthält $71,23 \%$ Chlor und musste dem Chlorgehalt entsprechend einen tiefen Schatten geben. Die $6 \mathrm{~mm}$ dicke Schicht des Salzes erwies sich für die X-Strahlen bei 21 Minuten Beleuchtungsdauer fast ganz undurchlässig. Nur nahe dem Boden des Gläschens ist die körnige Structur des Inhalts eben zu erkennen. Dies zeigt zugleich, wie die Energie der Strahlen nahe dem Centrum der Strahlenquelle sehr viel grösser ist, als nur wenig davon nach aussen.

Auch für die einfachste Chlorverbindung, die Salzsäure, war daher eine starke Undurchlässigkeit zu erwarten. Eine hochconcentrirte, wässrige Salzsäurelösung von wasserklarem Aussehen gab dementsprechend trotz des Wassergehaltes einen dunkelgraten Schatten. Die Säure enthielt etwa $30 \mathrm{Ge}$ wichtsprocente Salzsäuregas.

Alle diese Versuche zeigen nun, dass es das Atom der Halogene an sich jst, das den Schatten wirft, denn wo es auch in organischer oder anorganischer Bindung auftritt, stets geben die Halogene einen Schatten, der ungefähr dem Procentgehalt der Verbindung an diesen Elementen entspricht. Die Röntgen-Strahlen finden also die Halogene in den verschiedenen Stoffen heraus, einerlei in welcher Anordnung der Atome sie sich in diesen Körpern befinden. Sie ermöglichen somit eine Art qualitativer chemischer Analyse, zumal bei organischen Verbindungen, die zugleich nach der Intensität der Schatten eine gewisse quantitative Abschätzung gestattet.

Sehr markant trat mir diese Fähigkeit der X-Strahlen entgegen, als ich die Untersuchung über die Halogene auf die organischen Gewebe selbst und ihre wichtigsten Bestandtheile ausdehnte. Es lag nahe, z. B. die Schilddrüse wegen ihres Jodgehalts mit X-Strahlen aufzunehmen. Dem sehr geringen Jodgehalt entsprechend wurde aber kein markantes Resultat erhalten. Bei einem einjährigen Schwein erwies sich die Schilddrüsensubstanz durchlässiger, als das Muskelgewebo. Bei einem vierjährigen Ochsen hingegen fiel der Schatten der Schilddrüse entschieden dunkler aus, als der der Muskeln. Ob diese Differenzen nur auf einem verschiedenen Blutgehalt der untersuchten Gewebstheile beruhten, oder ob thatsächlich der Ochse in den vier Jahren seines Lebens schon mehr Jod in der Thyreoidea aufgespeichert hatte, als das einjährige Schwein, kann nur eine grössere, eingehende Versuchsreihe entscheiden, ebenso die Frage, ob fortgesetzte Jodfütterung die Durchstrahlbarkeit der Schilddrüse in nachweisbarem Grade verändert.

Um ein brauchbareres Resultat zu erlangen, wandte ich mich daher dem von Baumann aus der Schilddrüse rein dargestellten Thyrojodin zu. Das Thyrojodin soll nach der Elementaranalyse 
etwa $10 \%$ Jod enthalten. Der Schatten des Thyrojodins fiel nun über alles Erwarten schwach aus und zwar noch schwächer, als der einer 0,5\% igen Chlorwasserlösung. Man konnte daher mit Sicherheit behaupten, dass die Substanz nicht 10\% Jod enthielt. Eingezogene Erkundigungen ergaben denn auch, dass die Fabrik das reine Thyrojodin noch mit $97 \%$ Milchzucker versetzt, also einer Substanz, die als rein organisch in den X-Strahlen kaum einen Schatten werfen wird, und so in den Handel bringt. Das untersuchte Pulver enthielt also nur $3 \%$ Thyrojodin und, da dies zu $10 \%$ aus Jod besteht, nur $0,3 \%$ Jod. Für diesen Jodgehalt stimmt nun die Intensität des Schattens recht gut mit der des Chlorwassers überein. Die Verdünnung mit Milchzucker ist so gewählt, dass $1 \mathrm{~g}$ Thyrojodinmischung denselben Jodgehalt, wie $1 \mathrm{~g}$ Hammelschilddrüse hat. Im photographischen Bild fällt trotzdem der Schatten der Schilddrüse wesentlich intensiver aus, da die Drüse im Hämoglobin und dessen Eisengehalt noch eine weitere schwer durchlässige Componente besitzt.

Durch die grosse Güte des Herrn Prof. Baumann wurde mir später auch völlig reines Thyrojodin zur Verfügung gestellt. Lies ergab, wie zu erwarten, einen intensiveren Schatten, doch ist derselbe nicht so dunkel ausgefallen, wie ich wohl erwartet hatte. Allerdings war das Pulver nur sehr lose in das Gläschen eingefüllt und daher wenig dicht. $\mathrm{Ob}$ noch andere Momente dabei mitspielen, wird sich wohl erst mit Sicherheit entscheiden lassen, wenn einmal die rationelle Formel des Thyrojodins bekannt sein wird.

Eine Durchstrahlung des gewöhnlichen Meeresschwammes, der als Spongia usta nur $0,07-0,25 \%$ Jod enhält, liess keine stärkere Schattenbildung erwarten. Die zusammengepressten Schwammstücke ergaben ein schwaches Grau, das zudem noch zum Theil auf Rechnung der erdigen Basen kommt, an die das Jod im Badeschwamm gebunden ist Im Vergleich mit dem Kork, der das Gläschen verschliesst und der in den Bildern völlig verschwunden ist, erscheint immerhin der Schatten des Schwammes noch verhältinssmässig stark.

Besonders undurchläs-

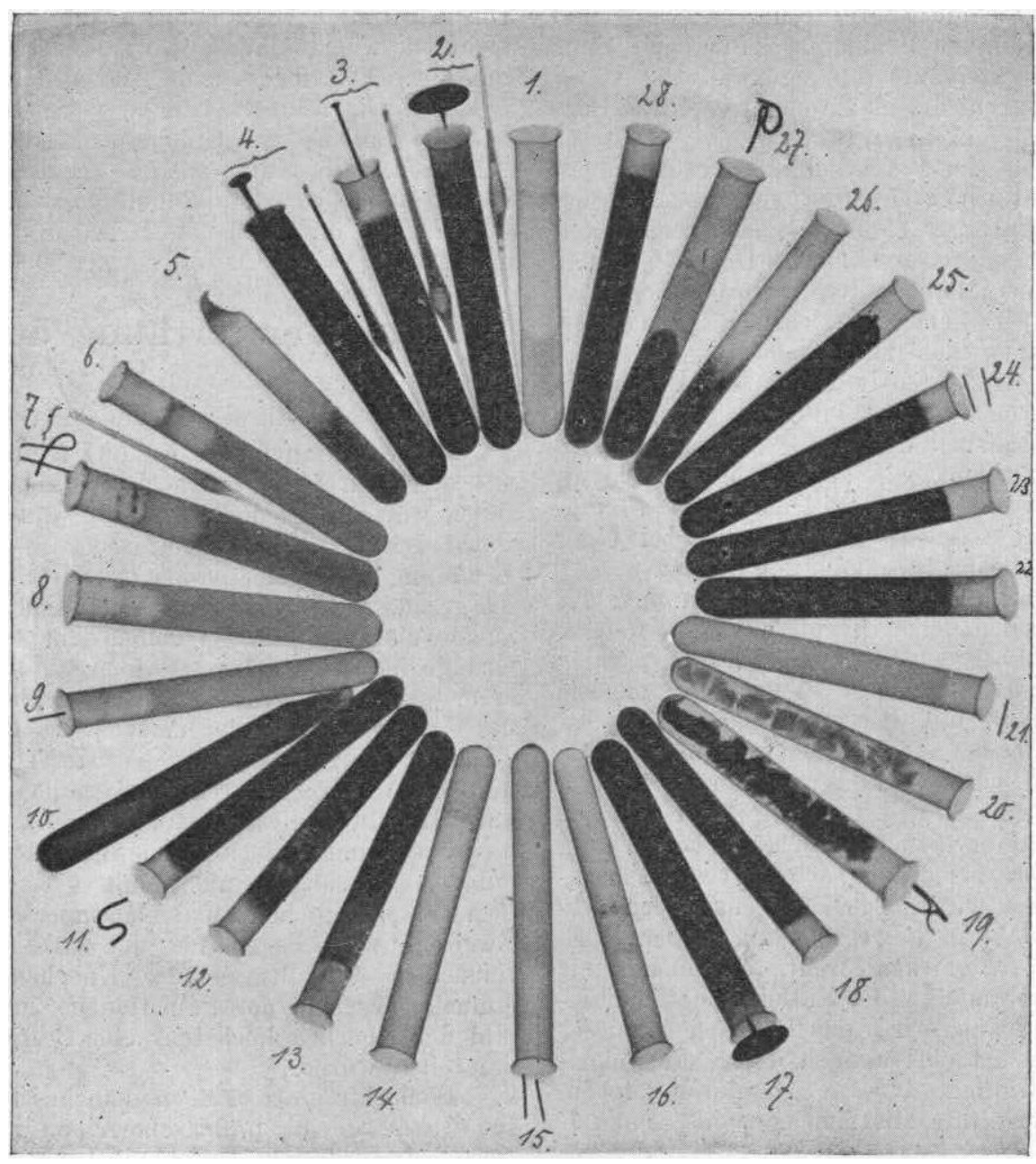

Tafel II. 1. Destillirtes Wasser. - 2. Chloroform (anch in Capillare). - 3. Chlorkohlenstoff, $\mathrm{CCl}_{4}$, (anch in Capillare). - 4. Bromoform (anch in Capillare). - 5. Salzsänre, ranchende. - 6. Eisen 10. 10. Rothe, ranchende salpetersant - 11. Thyrojodin rein. - 15. Terpentinöl - 16. Lampenrus 17. Chloroform, reinstes. - 18. Calciumoxyd. - 19. Kalinmoxydhydrat. - 20. Natrinmoxydhydrat - 24. Arsenire Sinre - 25. - 26. Rothe Phosphor. - 27. Gelber Phosphor. - 28. Chloralhydrat. nente zu setzen sein. Das Fluor würde sich dann in erwarteter Weise den übrigen Halogenen anreihen. Allerdings wirft auch das Calcium selbst schon einen starken Schatten, wie die Untersuchung Calciumoxyds zeigt, das $71,43 \%$ Calcium gebunden enthält

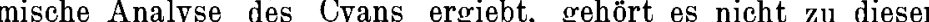
Obgleich das Cyankalium $40 \%$ Cyan (CN) enthält, also fast soviel um Beweise dass das Grade durchlässt.

Ich habe zur weiteren Controlle die wichtigsten Alkalimetalle Calciumoxyd wurde schon erwähnt, dass es mit seinen $71,43 \%$ Metall einen tief schwarzen Schatten in $6 \mathrm{~mm}$ dicker Schicht ergiebt. Das

Kaliumoxydhydrat mit $69,64 \%$ Kalium giebt in grösseren Stücken gleichfalls einen fast schwarzen Schatten, während dünnere Stückchen leicht durchscheinend sind. Ganz bedeutend viel durchlässiger ist das Natriumoxydhydrat mit 57,5\% Natrium, das auch in dicker Schicht nur graue, keineswegs schwarze Schatten gegeben hat.

Hätten die einzelnen Halogene ein wesentlich verschiedenes Absorptionsvermögen gegen die X-Strahlen gezeigt, so wäre eine verschiedene photographische Empfindlichkeit ihrer Silbersalze gegen diese Strahlen nicht unwahrscheinlich gewesen. Nach den gewonnenen Resultaten war eine solche kaum noch zu erwarten. Direkt auf diesen Punkt gerichtete Versuche ergaben, dass zunächst, wie für die Lichtstrahlen, so auch für die X-Strahlen das Bromsilber das empfindlichste ist. Das ausserordentlich grosse $\mathrm{Ab}$ sorptionsvermögen gerade des Broms dürfte aber doch vielleicht hierbei eine Rolle spielen.

In der Litteratur ist mir bisher nur eine Bemerkung aufgestossen, die einen kleinen Beitrag zu der hier sige Salze waren zu erwarten von einer Vereinigung der Halorene mit Metallen von hohem Atomgewicht, wie mit dem Gold (Atomgewicht 197), dem Platin (Atomgewicht 198) und Wismuth (Atomgewicht 210). Bei einer Verdünnung auf $1 \%$ hat das Goldchlorid noch einen dunkelgrauen Schatten gegeben, der auch in der Capillarröhre noch gleich deutlich ist. Das vier werthige Platin mit seinem relativ geringeren Metallgehalt hat in $1 \%$ iger Lösung einen etwas schwächeren Schatten geworfen. Das Eisenchlorid in $10 \%$ iger Lösung ergiebt einen Schatten, der seiner Tiefe nach zwischen dem der $1 \%$ igen Gold- und Platinlösung steht. Das niedrige Atomgewicht des Eisens (56) kommt darin zum Ausdruck. Da die Weichtheile des menschlichen Körpers, soweit sie in den Röntgenstrahlen einen Schatten werfen, diese Undurchlässigkeit vorwiegend dem Eisen des Hämoglobins und dem Chlorgehalt der Gewebsflüssigkeiten verdanken, abgesehen von den geringen Mengen an Alkalimetallen, war eine Prüfung des Eisenchlorids nicht ohne Interesse. Das Airol mit $24,8 \%$ Jod und $40,15 \%$ Wismuth hat sich als fast ganz undurchstrahlbar erwiesen.

Von dem vierten Halogen, dem Fluor, stand mir nur das Fluorcalcium zur Verfügung. Es besteht fast zur Hälfte, nämlich zu $48,72 \%$ aus Fluor. Der sehr intensive Schatten, den es ergab, dürfte daher zum Theil wohl auch auf Rechnung der Fluorcompo- behandelten Frage giebt. Im 25. Congress der Deutschen Gesellschaft für Chirurgie theilte Geissler (Berlin) mit, dass im Bild einer zwei Jahre lang beobachteten Ellenbogentuberkulose dunkle Flecke zu sehen waren, die man schliesslich als eingespritztes Jod $\mathrm{zu}$ deuten in der Lage war.

Die grosse Aehnlichkeit, welche Halogene und Metalle den X-Strahlen gegeniiber besitzen, durch die auch ihre Bezeichnung als Metalloide eine neue Berechtigung gewinnt, äussert sich aber auch noch in ganz anderer Weise. Bekanntlich beschleunigen die Röntgen-Strahlen die Entladung elektrisch geladener Körper. Diese Beschleunigung ist aber in verschiedenen Gasen verschieden gross. J. J. Thomson und Mc. Clelland ${ }^{1}$ ) fanden nun, dass die Entladung in Quecksilberdämpfen am schnellsten ist. Ueber alles Erwarten gross war sie aber auch im Gas der Halogene. Auch hier stellen sich also die Halogene den Metallen im Verhalten gegen X-Strahlen fast gleich.

Im Anschluss an die Halogene habe ich noch eine Anzahl anderer Metalloide untersucht, die zum Theil im menschlichen Körper eine wichtige Rolle spielen, wie den Schwefel und Phosphor. Gelber und rother Phosphor ist in hohem Grade undurchlässig

I) Nature 1896, Vol. LIII, S. 581 
für Röntgenstrahlen, der gelbe noch etwas mehr als der rothe. Erst in dünner Schicht werden sie in geringem Maasse durchstrahlbar. Ebenso ergab auch der Schwefel als Schwefelblumen, als präcipitirter und aus dem präcipitirten zusammengeschmolzener Schwefel ein sehr starkes Absorptionsvermögen. Das liess erwarten, dass der Schwefelkohlenstoff, der eine wasserklare Flüssigkeit darstellt, ein weiterer im Licht völlig durchsichtiger Körper sein möchte, der, mit Röntgenstrahlen beleuchtet, einen tiefen Schatten geben müsste, da der Schwefelkohlenstoff ja nur aus $15,79 \%$ völlig durchlässiger Kohle, aber aus 84,21\% durchlässigem Schwefel besteht. Auch hier entsprach der Versuch vollständig der theoretischen Voraussetzung. In $6 \mathrm{~mm}$ dicker Schicht war der Schatten des Schwefelkohlenstoffes tief schwarz, erst in viel dünnerer Lage wurde er tief dunkelgrau. - Der dunkele Schatten der Knochen ist also nicht bloss durch Calcium, sondern zugleich auch durch den Phosphorgehalt des phosphorsauren Kalkes bedingt. Reines Arsen und arsenige Säure, reines Antimon und Antimonoxyd lassen selbst in dünnen Schichten kaum eine Spur der X-Strahlen hindurch. Die Borsäure hingegen ist fast völlig durchlässig, wie es bei dem sehr niedrigen Atomgewicht des Bors (11) zu erwarten war, sie enthält $77,42 \%$ Bor.

Kurz erwähnen will ich noch den Befund einiger Verbindungen der schweren Metalle, die therapeutisel öfter verwandt werden und dann bei Aufnahme der kranken Theile mit Röntgenstralılen leicht zu Irrthümern Veranlassung geben können. Das Bleipflaster und Heftpflaster ist infolge des Bleigehaltes undurchlässig und selbst in so dünnen Lagen, wie beim gestrichenen Heftpflaster, bildet es sich noch durch einen deutlichen Schatten ab. Der Höllenstein in Substanz giebt ein intensiv schwarzes Bild. Mit Höllenstein geschwärzte Epidermis kommt im Photogramm nicht zum Ausdruck, wenn die Epidermislage der photographischen Platte parallel läuft. Steht sie hingegen senkrecht zu der Gelatineschicht, dann können so starke Schatten auftreten, dass leicht kleine Fremdkörper im Gewebe vorgetäuscht werden, wie ich das bei einer Schussverletzung an der Hand beobachten konnte.

Schliesslich sei ein physikalischer Versuch noch erwähnt, der zwar ausser Zusammenhang mit dem Thema steht, aber eine weitere Differenz der X-Strahlen gegenüber den Licht- und Wärmestrahlen ergiebt. Die Wirkung der X-Strahlen auf die photographische Platte steht in vollem Einklang mit der Wirkung der Lichtstrahlen. Es fragte sich, ob noch andere Lichtwirkungen durch die X-Strahlen zu erzielen sind. Die Vereinigung von Chlor- und Wasserstoffgas zu Salzsäure ist durch die Bestrahlung mit X-Strahlen bisher noch nicht geglückt. Ich untersuchte noch die Einwirkung auf das Crookes'sche Radiometer, das durch Licht-, event. Wärmestrahlen in Umdrehung versetzt wird. Die Röntgenstrahlen vermögen das kleine Rädchen nicht in Bewegung zu setzen. Das Rädchen wird zwar einen kurzen Moment gedreht, aber immer nur so weit, bis der nächste Aluminiumflügel der Kathode der Vacuumröhre direkt gegenübersteht. Dann wird das Rädchen in dieser Stellung festgehalten. Dies erklärt sich einfach durch die Polarisationswirkung von Seiten der Kathode auf das Aluminiumrädehen.

Fasse ich die erhaltenen Resultate zusammen, so hat sich folgendes ergeben:

1. Die Halogene Chlor, Brom und Jod sind in reinem Zustand für die Röntgenstrahlen in hohem Maasse undurchlässig. Sie ähneln darin sehr den Metallen und es gewinnt dadurch ihre Bezeichnung als Metalloide eine neue Berechtigung.

2. Diese Undurchlässigkeit ist an das Atom der Haloide gebunden und nicht etwa eine Folge der Atomgruppirung im Molekül.

3. Infolge dessen zeigen auch alle chemischen Verbindungen der Halogene eine Undurchstrahlbarkeit für X-Strahlen, die dem Procentgehalt der Verbindung an Halogenen entspricht. Die Röntgenstrahlen ermöglichen somit für Stoffe, die in ihrer Verbindung nur ein schattengebendes, chemisches Element enthalten, eine gewisse, wenn auch beschränkte qualitative und selbst quantitative chemische Analyse und lassen in manchen Fällen Verfälschungen leicht und sicher nachweisen.

4. Es giebt eine Reihe wasserklarer, für Licht völlig durchlässiger Flüssigkeiten, die wegen ihres hohen Halogengehaltes für X-Strahlen fast ganz undurchgängig sind, und daher eine Art Filter für diese Strahlen darstellen. Hierher gehört die Salzsäure, das wasserklare Chloroform, der flüssige Chlorkohlenstoff $\mathrm{CCl}_{4}$ und vor allem das flüssige, wasserklare Bromoform. Das Bromoform giebt selbst in dünnster Schicht einen intensiv schwarzen Schatten, der sich von dem Schatten einer gleich dicken Metall-, z. B. Messingschicht kaum unterseheidet.

5. Das Cyan ist trotz seiner chemischen Aehnlichkeit mit den Halogenen doch für die X-Strahlen leicht durchlässig, wie es auch seine chemische Zusammensetzung als rein organischer Körper erwarten liess.
6. Die vier Grundelemente der organischen Verbindungen, der Kohlenstoff, Wasserstoff, Sauerstoff und Stickstoff sind für X-Strahlen fast völlig durchlässig. Nur der Stickstoff absorbirt einen etwas grösseren Antheil der Strahlen.

7. Der Schatten, den thierische Weichtheile werfen, beruht neben dem Eisengehalt des Hämoglobins und der Alkalimetalle zum grossen Theil auf ihrem Chlorgehalt

8. Von den übrigen Metalloiden werfen Plosphor und Schwefel, noch mehr Arsen und Antimon intensive Schatten, hingegen viel weniger das Bor. Aucl der Schwefelkohlenstoff ist eine wasserklare Flüssigkeit, die von den X-Strahlen nur sehr wenig hindurchtreten lässt und daher in ihnen einen dunklen Schatten wirft.

9. Das Natrium ergiebt gegenüber dem Kalium und Calcium nur einen verhältnissmässig geringen Schatten.

10. Eine weitere Aehnlichkeit zwischen den Metallen und Halogenen gegenüber den Röntgenstrahlen zeigt sich in folgendem: Die X-Strahlen beschleunigen die Entladung elektrisclier Körper. Aehnlich wie in Quecksilberdämpfen ist diese Beschleunigung auch im Gas der Halogene eine sehr bedeutende.

(11. Die Röntgenstrahlen vermögen nicht, wie die Licht- und Wärmestrahlen, das Crookes'sche Radiometer in Umdreliung zu versetzen. Die Katlıode der Vacuumröhre dreht nur den ihr zinächst stehenden Aluminiumflügel so weit, bis er ihr genau gegenüber stelit, infolge der Polarisation, die sie auf das Aluminium ausübt.) 